\title{
PD-related stresses in the bulk dielectric and their evaluation
}

\section{Pedersen, Aage; Crichton - Fratrådt, George C; McAllister, lain Wilson}

\section{Published in:}

Proceedings of the Conference on Electrical Insulation and Dielectric Phenomena

Link to article, DOI:

10.1109/CEIDP.1993.378927

Publication date:

1993

Document Version

Publisher's PDF, also known as Version of record

Link back to DTU Orbit

\section{Citation $(A P A)$ :}

Pedersen, A., Crichton - Fratrådt, G. C., \& McAllister, I. W. (1993). PD-related stresses in the bulk dielectric and their evaluation. In Proceedings of the Conference on Electrical Insulation and Dielectric Phenomena (pp. 474480). IEEE. https://doi.org/10.1109/CEIDP.1993.378927

\section{General rights}

Copyright and moral rights for the publications made accessible in the public portal are retained by the authors and/or other copyright owners and it is a condition of accessing publications that users recognise and abide by the legal requirements associated with these rights.

- Users may download and print one copy of any publication from the public portal for the purpose of private study or research.

- You may not further distribute the material or use it for any profit-making activity or commercial gain

- You may freely distribute the URL identifying the publication in the public portal 
PD-RELATED STRESSES IN THE BULK DIELECTRIC

AND THEIR EVALUATION
A. Pedersen
G. C. Crichton and I. W. McAllister

Physics Department

Building 309

Electric Power Engineering Department Building 325

The Technical University of Denmark DK-2800 Lyngby, Denmark

\section{INTRODUCT ION}

The conventional method of detecting partial discharges in voids consists of recording potential or current transients at the terminals of the system. These transients occur due to the electricification by electrostatic induction of the detecting electrode [1]. The primary field source which gives rise to this phenomenon is the surface charge distribution on the wall of the void, brought about by partial discharge activity. Electricification by induction would not arise if the charges within the void did not distort the field external to the void.

A direct calculation of this external field distortion would be possible if we knew the location of the void and the distribution of charges within the void. In general, however, this knowledge is not available. Nevertheless, we can obtain reliable information on this important problem from a knowledge of the charge induced on a detectingelectrode/system-terminal.

Viewed from the detecting electrode, the charges deposited on the wall of the void (both +ve and -ve) can be considered to a first approximation as an electric dipole configuration of dipole moment $\vec{\mu}$. This is justifiable, as the net charge within the void remains zero. The Poissonian induced-charge $q(t)$ which arises from such a dipole is given by [2]

$q(t)=-\vec{\mu}(t) \cdot \vec{\nabla} \lambda$

The Poissonian induced-charge is that part of the charge on the electrode which together with the charge distribut- 
ion on the wall of the void is the source for the Poissonian part of the field distribution [1]. $\lambda$ is a dimensionless positive scalar function which relates the charge in the dielectric to the charge induced on the detecting e1ectrode; it is simply a proportionality factor. The $\lambda$ function is a solution of the general Laplace equation for the following boundary conditions. $\lambda=1$ at the detecting electrode and $\lambda=0$ at all other electrodes [2].

If we measure $q(t)$, and know the location and shape of the void, the dipole moment of the charges within the void can be obtained from (1). This requires a knowledge of $\lambda$. Owing to the presence of the void, a direct calculation of $\lambda$ becomes cumbersome. We can simplify the procedure by considering the value which $\lambda$ would attain at a location identical to that of the void, but with the entire dielectric system now assumed void-free. Let us denote this function by $\lambda_{0}$. The two functions are related by [3]

$\vec{\nabla} \lambda=h \vec{\nabla} \lambda_{0}, \quad$ with $1<h<\varepsilon_{r}$

where $\varepsilon_{r}$ is the relative permittivity of the bulk dielectric in which the void is embedded. If we restrict this concept to voids of simple form, such as spheroids, and to isotropic dielectrics, then $h$ is a scalar. Generally however $h$ will be a tensor. For a spherical void $h$ is given by

$h=\frac{3 \varepsilon_{r}}{1+2 \varepsilon_{r}}$

We note that $h$ depends simply on $\varepsilon_{r}$ and the shape of the void.

The relationship between Poissonian induced-charge and space-charge dipole-moment may thus be written in the form

$q(t)=-\vec{\mu}(t) \cdot h \vec{\nabla} \lambda_{0}$

In the present study, we quantify the external field perturbation associated with the dipole moment, and discuss its relevance as a contributing factor towards the failure in electrical insulation capability of a solid dielectric. 
FIELD PERTURBATION IN BULK DIELECTRIC

The nature of the field perturbation in the bulk dielectric can be assessed through a simple model. Consider a point dipole $\vec{\mu}$ located at the centre of a spherical void in an extended dielectric with constant permittivity. With reference to Fig.1, the potential $\Delta \phi(r, a)$ associated with $\vec{\mu}$ at a point $\mathrm{P}$ outside the void, i.e., for $r \geq R$, where $R$ is the radius of the spherical void, is given by

$\Delta \phi(r, \alpha)=\frac{\mu}{4 \pi \varepsilon_{0}} \frac{3}{\left(1+2 \varepsilon_{r}\right)} \frac{\cos \alpha}{r^{2}}$

where $\alpha$ is the angle between the direction of $\vec{\mu}$ and the line from the dipole to the point in question. To this potential should be added the perturbation from the induced charge on the electrode. However this contribution can be neglected since we are only interested in the field in the vicinity of the void.

The field strength $\overrightarrow{\Delta E}$ at $\mathrm{P}$ can be considered as the sum of two components. A component $\overrightarrow{\Delta E}$, orientated in the direction of $\vec{r}$, and $\overrightarrow{\Delta \vec{E}} 2$ directed at right angles to $\vec{r}$ in the plane containing $\vec{r}$ and $\vec{\mu}$. These components are given by

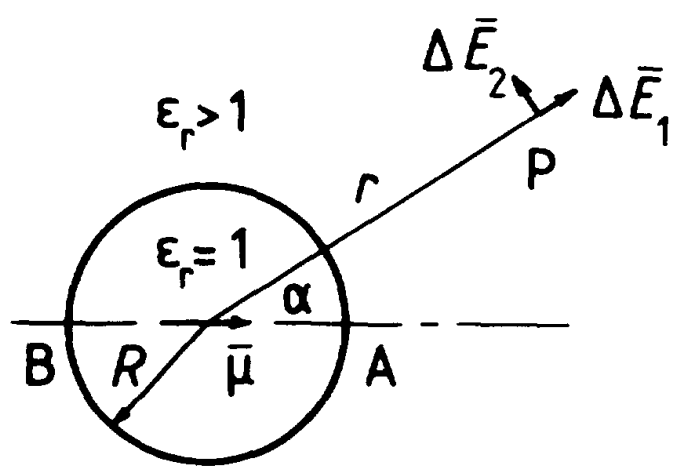

Fig.1. External field distortion model. 


$$
\Delta E_{1}=-\frac{\partial \Delta \phi}{\partial r}=\frac{\mu}{4 \pi \varepsilon_{o}} \frac{6}{\left(1+2 \varepsilon_{r}\right)} \frac{\cos \alpha}{r^{3}}
$$

and

$$
\Delta E_{2}=-\frac{1}{r} \frac{\partial \Delta \phi}{\partial \alpha}=\frac{\mu}{4 \pi \varepsilon_{\mathrm{o}}} \frac{3}{\left(1+2 \varepsilon_{\mathrm{r}}\right)} \frac{\sin \alpha}{r^{3}}
$$

This field perturbation attains its maximum value in the solid dielectric at $\mathrm{A}$ and $\mathrm{B}$, see Fig.1, i.e. for $r \rightarrow R$ and $\alpha=0$ and $\alpha=\pi$.

On introducing the void volume $\Omega=\frac{4}{3} \pi R^{3}$, the maximum field strength inside the solid dielectric at $A$ and $B$ can be expressed as

$\Delta \vec{E}_{\mathrm{A}}=\Delta \vec{E}_{\mathrm{B}}=\frac{2 \vec{\mu}}{\varepsilon_{\mathrm{o}}\left(1+2 \varepsilon_{\mathrm{r}}\right) \Omega}$

$\Delta \vec{E}_{\mathrm{A}}, \Delta \vec{E}_{\mathrm{B}}, \vec{\mu}$ and the externally applied field are all coparallel.

Recalling the value of $h$ for a spherical void, we can rewrite (8) as

$\Delta \vec{E}_{\mathrm{A}}=\frac{2 h \vec{\mu}}{3 \varepsilon_{\mathrm{o}} \varepsilon_{\mathrm{r}} \Omega}$

It is important to observe that, with respect to partial discharge evaluation, the concept of $\vec{\mu}$ is derived from a multipole expansion [2], and thus $\vec{\mu}$ cannot be used to estimate the actual field distribution within the void.

\section{Partial Discharges and Dipole Moments}

In the following we continue to restrict our analysis to small spherical voids such that the internal field can be considered to be effectively uniform prior to the first discharge. A partial discharge can develop when the applied field within the void reaches the inception value $E_{i}$. The ensuing discharge results in a buildup of charge on 
the surface of the spherical void that subsequently reduces the field within the void. The discharge is ultimately quenched when the maximum value of the internal field is reduced to the 1 imiting value $E_{\ell} ; i . e$. the field value below which ionization growth is impossible for the void gas pressure in question.

To simplify the analysis we will assume that the internal field remains uniform and that the entire spherical volume is active in the discharge. This makes it possible to quantify the dipole moment of the surface charge distribution in the void. Even if these assumptions are not necessarily fulfilled in practice, the general conclusions which can be drawn from the analysis will remain valid.

The above approach was adopted previously by the authors [3]. From that study the dipole moment of the surface charges deployed by a single discharge in a spherical void may be expressed as

$\vec{\mu}=\frac{3}{h} \Omega \varepsilon_{0} \varepsilon_{r}\left(\vec{E}_{i}-\vec{E}_{\ell}\right)$

Upon substituting (10) into (9) we obtain

$\Delta \vec{E}_{A}=2\left(\vec{E}_{i}-\vec{E}_{\ell}\right)$

This value represents the initial value of the field distortion in the bulk of the dielectric at $A$ (and $B$ ), see Fig. 1, as a result of the first discharge. It should be noted that the distortion remains at this value even after the discharge is quenched, as it is directly 1 inked with the charges which have been deposited on the surface of the void.

For a spherical void of volume $1 \mathrm{~mm}^{3}$ filled with air at a pressure of $0.1 \mathrm{MPa}$, the streamer criterion [3] yields the following value for $E_{i}$ : viz.,

$E_{i}=4.3 \mathrm{kV} / \mathrm{mm}$, with $E_{\ell}=2.4 \mathrm{kV} / \mathrm{mml}$

Hence, the very first discharge could generate a field distortion within the solid dielectric, in the proximity of the void, of the order of 
$\Delta E_{\mathrm{A}}=\Delta E_{\mathrm{B}}=4 \mathrm{kV} / \mathrm{mm}$

This is a value of field stress which must be taken seriously in insulation design, especially if we bear in mind that this figure might increase by several fold. This would be the case if the first discharge were followed by a series of similar discharges in the same direction, as each successive discharge would additively effect the distortion of the field. Moreover, the applied field, which promotes the partial discharge development within the void, and the ensuing dipole field are, external to the void, coparallel. Consequently, in the proximity of the void, partial discharging will result in a potentially-damaging field-enhancement buildup within the bulk dielectric.

\section{DISCUSSION}

Through the application of electromagnetic field theory to the subject of partial discharges, it is shown that discharging in a void generates large field distortions within the bulk dielectric, in the proximity of the void. Such inherent over-stressing of a dielectric could be the effect which triggers the onset of electrical treeing and other damaging processes, and which subsequently precipitates the breakdown of the insulation.

The above insight cannot be gained from the capacitancemodel approach, an approach upon which partial discharge detection procedures and standards of today are based. According to this latter approach, the electrical signals which can be detected at the system terminals are to be associated purely with changes in system capacitance. The actual form of the internal field distribution is, in the capacitive model, viewed as unperturbed and the field distribution is Laplacian.

The basic parameter in our field-theoretical approach is

the dipole moment $\vec{\mu}$, which relates the surface charges accruing within the void following discharge activity. In the present study this parameter is evaluated by relating the wall-charge internal-field to the field strength required to suppress the discharge growth. However, in practice, $\vec{\mu}$ could be evaluated if the Poissonian inducedcharge $q$ on the electrode/system-terminal were determined from measurement [1]. 
As has been shown previously [2], proportionality exists between $\vec{\mu}$ and $q$ as expressed in (1). A knowledge of $\lambda$, and of the function $q(t)$ from practical measurements, would

allow the variation of $\vec{\mu}$ with time to be determined at any location within the dielectric. In particular, a knowledge of the maximum value of $\vec{\mu}(t), \vec{\mu}_{m}$, for a sequence of discharges, would enable maximum stress-levels to be estimated. Thus, from such measurements, a sound basis for the correct dimensioning of the insulation could be achieved.

\section{CONCLUSION}

Using a field-theoretical approach, the existence of a substantial field enhancement in a solid dielectric, near to a void undergoing partial discharging, is shown to be an inherent consequence of such discharge activity.

It has not escaped our notice that such a localized stress enhancement has very considerable practical implications. For example, if there were a train of partial discharge events per power frequency cycle, then, during each half period, these events would lead to accumlative stress levels within the solid dielectric. We thus conclude that stress cycling of this nature is the mechanism which is responsible for the initiation of electrical treeing and other insulation-degrading processes, and which ultimately brings about the complete rupture of the solid dielectric.

\section{REFERENCES}

[1] A. Pedersen, G.C. Crichton and I.W. McAllister, "Partial Discharge Detection: Theoretical and Practical Aspects", International Conference on Partial Discharge, Canterbury, 1993.

[2] A. Pedersen, G.C. Crichton and I.W. McAllister, "The Theory and Measurement of Partial Discharge Transients", IEEE Trans. Elect. Insul., Vo1.26, pp.487-497 1991.

[3] G.C. Crichton, P.W. Karlsson and A. Pedersen, "Partial Discharges in Ellipsoidal and Spheroidal Voids", IEEE Trans. Elect. Insu1., Vol.24, pp.335-342, 1989. 